\title{
O ENVOLVIMENTO DO PAI NO PROCESSO \\ DESENVOLVIMENTAL DOS FILHOS: UMA ABORDAGEM INTERGERACIONAL
}

\author{
Albino Lima \\ Rui Serôdio ${ }^{2}$ \\ Orlanda Cruz ${ }^{3}$
}

Resumo: Neste estudo exploramos a relação entre as formas de envolvimento do pai contemporâneo no processo desenvolvimental dos filhos em idade escolar e a representação que o pai tem acerca do envolvimento do seu próprio pai, ao longo da infância e adolescência. Considerámos duas formas de envolvimento paterno: tempo despendido em Interacção e em Acessibilidade, avaliadas a partir do Guião de Entrevista Interacção e Acessibilidade. Foi ainda utilizada a escala ENVOLVE que se reporta à representação do pai acerca do envolvimento do seu próprio pai. Participaram 189 pais e 407 crianças (8-10 anos). Os resultados, entre outros aspectos, mostram que, quanto mais positiva é a representação do envolvimento acerca do seu próprio pai, mais o pai interage com a criança. Isto verifica-se, particularmente, na representação que o adulto tem do envolvimento do seu pai nas dimensões Apoio Emocional e Estimulação e Partilha de Tarefas com a mãe. Discutimos estes resultados em termos das continuidades e descontinuidades intergeracionais, enquanto determinantes do envolvimento paterno.

Palavras-chave: envolvimento paterno, interacção, história desenvolvimental, intergeracional

Falher Involvment in the Offspring Developmental Process: An Intergenerational Approach (Abstract): The present study addresses the relations between the involvement of the contemporary father in the developmental process of their school age offsprings, and the representation of his own father involvement throughout childhood and adolescence. Two forms of father involvement were considered: Amount of time spent in Interaction and in Availability, which was measured using the Interaction and Availability Interview Guide and adults' retrospective representation of their own father involvement, which was assessed using the

\footnotetext{
${ }^{1}$ Faculdade de Psicologia e de Ciências da Educação, Universidade do Porto

2 Faculdade de Psicologia e de Ciências da Educação, Universidade do Porto

${ }^{3}$ Faculdade de Psicologia e de Ciências da Educação, Universidade do Porto 
Father Involvement Scale. The study was conducted with 189 fathers and 407 children (8-10 years-old). Overall, results showed that the more positive the father's representation of his own father involvement, especially in what concerns Emotional Support and Stimulation and Sharing of Domestic Work with the mother, the more he interacted with the child. These results are discussed in terms of the intergenerational continuities and discontinuities as determinants of the involvement of the father.

Keywords: father involvement, interaction, developmental history, intergenerational

\section{Introdução}

A Psicologia do Desenvolvimento tem contribuído de forma fundamental para transformar o modo como os investigadores têm considerado o envolvimento paterno enquanto objecto de estudo (Camus \& Frascarolo, 2003), realçando as formas, factores e consequências desse mesmo envolvimento.

Este interesse pelo envolvimento do pai no processo desenvolvimental dos filhos aparece associado a transformações sociais significativas, como sejam, as (novas) configurações de família, a participação maciça da mulher no mercado de trabalho, a redução da natalidade, o aumento exponencial do número de divórcios e as questões de regulação do exercício do poder paternal, as mudanças na definição de papel parental ou, ainda, as políticas de apoio à família e o debate sobre o desenvolvimento e bem-estar da criança.

Apesar de toda a discussão acerca do constructo envolvimento paterno (cf. Hawkins, Bradford, Palkovitz, Christiansen, Day, \& Call, 2002), a investigação tem sido marcada por uma base conceptual de referência: o modelo tripartido de envolvimento paterno proposto originalmente por Lamb, Pleck, Charnov \& Levin (1987). Este modelo tem-se mostrado de grande utilidade e de manifesto valor heurístico, distinguindo três formas de envolvimento paterno: (1) Interacção: diz respeito à interacção directa entre o pai e a criança; (2) Acessibilidade: o pai pode, ou não, envolver-se directamente em momentos de interacção mas está disponível para o filho; (3) Responsabilidade: o envolvimento do pai implica a assumpção de responsabilidades pelo bem-estar, segurança e satisfação das necessidades da criança. Neste estudo vamos centrar-nos sobre as duas primeiras formas de envolvimento.

Diversos autores (e.g. Ahlberg \& Sandnabba, 1998; Parke, 1995, 2000) têm proposto modelos que enfatizam a história desenvolvimental do pai enquanto factor determinante no envolvimento paterno. De acordo com Pleck (1997), um dos aspectos que na história desenvolvimental do pai recebe maior atenção diz respeito ao envolvimento do pai com o seu próprio progenitor. 
Com efeito, o pai tende a seguir o modelo de parentalidade do seu próprio pai mais do que o da sua mãe (Losh-Hasselbart, 1987) e muitas vezes perspectiva o seu futuro enquanto pai em função das experiências positivas ou negativas de envolvimento com o seu próprio pai (Marsiglio, Hutchinson, \& Cohan, 2000). A propósito deste tópico, os estudos apresentam genericamente duas hipóteses: (1) a modelagem (quer o envolvimento seja elevado ou baixo) e (2) a compensação (nas situações de baixo envolvimento).

A hipótese da modelagem tem por base a teoria da aprendizagem social (Parke, 1995) e é suportada por vários estudos (e.g. Palkovitz, Marks, Appleby, \& Holmes, 2003; Pleck, 1997, 2004). Neste caso, os progenitores constituem um modelo para os pais havendo deste modo continuidade intergeracional nas formas de envolvimento paterno. De acordo com os autores, a modelagem constata-se, quer em indivíduos cujos pais se envolveram de forma significativa no seu processo de socialização, quer em indivíduos em que tal não sucedeu.

A hipótese da compensação é fundamentada pela associação entre alto envolvimento e baixa qualidade percebida desse envolvimento (Volling \& Belsky, 1992). Segundo Ahlberg e Sandnabba (1998) muitas investigações constataram a tendência dos homens para se comportarem de forma oposta à dos seus próprios pais. Estudos qualitativos sugerem que muitos homens não consideram os seus próprios pais como modelos positivos e querem ser melhores exemplos para os seus filhos do que os pais o foram para eles (Marsiglio, Amato, Day, \& Lamb, 2000; Pleck, 1997), envolvendo-se activamente no processo de socialização da criança (Lamb, 2000). Os homens cujos pais se envolveram ao longo do seu desenvolvimento, revelam-se também mais envolvidos na socialização dos seus filhos. Assumem mais responsabilidades para com eles, mostram-se mais calorosos, e mais próximos no acompanhamento dos comportamentos e actividades dos filhos (Hofferth \& Sandberg, 2001).

O sexo da criança é um factor relevante a considerar e vários estudos sugerem a sua influência ao nível do envolvimento paterno (e.g. Wood \& Repetti, 2004). Genericamente, as investigações revelam que os pais envolvem-se mais com os filhos do que com as filhas (McBride, Schoppe, \& Rane, 2002; Raley \& Bianchi, 2006), despendendo mais tempo em interacção com um rapaz (Fuligni \& Brooks-Gunn, 2002).

\section{Enquadramento e objectivos do estudo}

Este trabalho insere-se no âmbito de uma investigação mais ampla acerca das formas, factores e consequências do envolvimento do pai no processo desenvolvimental dos filhos em idade escolar (Lima, 2009). O objecti- 
vo central deste estudo é o de explorar a relação entre o tempo de envolvimento paterno percepcionado pelas crianças, com a representação retrospectiva que o pai faz acerca envolvimento do seu próprio pai, ao longo da infância e adolescência. Explora-se, ainda, em que medida o sexo da criança é um factor moderador da relação entre aquelas variáveis.

\section{Método}

\section{Participantes}

Participaram no estudo 407 crianças do $3^{\circ}$ ano de escolaridade (sexo masculino, $n=206$; sexo feminino, $n=201$ ) de 23 turmas distribuídas por 10 escolas do $1^{\circ}$ Ciclo do Ensino Básico do Centro da Área Educativa do Porto. A idade dos participantes varia entre os 8 e os 10 anos $(M=8.17, D P$ $=0.47$ ). Participaram também no estudo os pais dos alunos, obtendo-se uma amostra de 162 homens. A média de idades destes pais é de 39.49 anos (DP $=5.80$; idades entre 25 e 58 anos). Em termos de escolaridade verificamos que os pais frequentaram, em média 10.40 anos escolares $(D P=4.72)$.

\section{Instrumentos}

Foi utilizado o Guião de Entrevista Interacção e Acessibilidade GUIA (Lima, 2009). Basicamente, o GUIA é uma entrevista semi-estruturada na qual as crianças procuram descrever, de forma tão pormenorizada quanto possível e com o auxílio do entrevistador, toda a sua rotina e actividades desenvolvidas ao longo do dia. Permite obter informação acerca do quotidiano da criança focando as actividades que ela realiza, quando, onde e com quem. Os participantes reportam-se a um dia de semana (o dia anterior ao da entrevista) e ao último dia de fim-de-semana (Sábado ou Domingo). No consequente processo de codificação são considerados períodos de 10 minutos, resultando em 144 períodos de tempo por cada rotina diária. Em cada entrevista é considerado para análise o período de vigília dos participantes.

A partir do GUIA é analisado o tempo de envolvimento entre o pai e a criança, tendo em conta o tempo despendido em Interacção e em Acessibilidade. A primeira diz respeito a situações de interacção directa entre o pai e a criança; na segunda o pai pode, ou não, envolver-se directamente na interacção, mas continua disponível (pelo menos fisicamente) para a criança.

Na Escala de Envolvimento Paterno - ENVOLVE (Lima, Serôdio, \& Cruz, 2008), é solicitado aos indivíduos que façam uma apreciação retrospectiva a propósito do envolvimento do seu próprio pai, ao longo da infância e adolescência. Este instrumento é composto por 40 itens, apresentando uma 
estrutura factorial com as seguintes dimensões: (1) Apoio Emocional e Estimulação - AEE, (por exemplo, "O meu pai era afectuoso comigo"); (2) Escola e Actividades Sociais - EAS, (por exemplo, "O meu pai assistia às actividades escolares em que eu participava"); (3) Sustento e Orientação Moral - SOM, (por exemplo, "O meu pai sustentava-nos financeiramente"); (4) Punição - PUN, (por exemplo, "O meu pai batia-me"); e (5) Partilha de Tarefas - PT, (por exemplo, "O meu pai cozinhava em casa"). A resposta às questões é dada numa escala de 5 pontos, com a seguinte rotulagem: "Nunca" (=0), "Raramente" (=1), "Algumas vezes" $(=2)$, "Muitas vezes" (=3), "Sempre" (=4). A ACP sobre estes itens revela uma estrutura factorial correspondente aos cinco factores enunciados (variância explicada, 63.89\%), os quais, apresentam valores de consistência interna entre moderados a elevados ( $\alpha$ de Cronbach entre 0,73 e 0,97). O valor de consistência interna da escala total é de $\alpha=.95$.

\section{Procedimento}

Solicitou-se aos órgãos de direcção das diversas escolas autorização para levarmos a cabo uma investigação com os alunos ( 8 a 10 anos de idade) que frequentassem o $3^{\circ}$ ano do $1^{\circ}$ ciclo do ensino básico. Posteriormente a investigação implicava também a participação dos pais dos referidos alunos. Em todas as escolas foram prestadas as informações que os órgãos de direcção entenderam convenientes, obtendo-se também o consentimento dos professores e dos pais ou encarregados de educação das crianças que voluntariamente decidiram participar no estudo. Em todos estes momentos procurámos que as tomadas de decisão dos diferentes intervenientes fossem informadas.

A administração do Guião de Entrevista - Interacção e Acessibilidade, foi feita utilizando o formato de entrevista individual com cada uma das crianças participantes. Neste caso, o entrevistador procurava que cada criança descrevesse de forma detalhada a sua rotina diária, seguindo as orientações do GUIA. As entrevistas decorreram numa sala ou gabinete disponibilizado pela escola, em ambiente tranquilo, procurando que os participantes se sentissem confortáveis e salvaguardando a sua privacidade. Para cada diário, o tempo médio de duração de preenchimento foi de 15 minutos.

A escala de Envolvimento Paterno (ENVOLVE) diz respeito à representação do pai acerca do envolvimento do seu próprio pai. Neste sentido, pretendia-se obter a participação dos pais dos alunos participantes. Num primeiro momento foi enviado, para todos os pais ou encarregados de educação das crianças, documentação em que se fazia o enquadramento e explicava os objectivos do estudo, solicitando o envolvimento dos pais, enquanto respondentes, a um questionário acerca das formas de envolvimento paterno, 
ao longo da infância e adolescência. Os pais que decidiram participar receberam um envelope contendo o questionário. As respostas foram devolvidas no envelope fechado garantindo-se a confidencialidade das respostas.

\section{Resultados}

Nesta secção, para além de uma abordagem descritiva sobre o tempo despendido pelo pai em Interacção e em Acessibilidade com a criança, iremos analisar em que medida o tempo de envolvimento paterno varia em função da representação do envolvimento do seu próprio pai, ao longo da infância e adolescência. Nesta análise iremos ainda verificar em que medida o Sexo da criança é um factor moderador da relação entre as variáveis atrás referidas.

Tempo de Interacção e de Acessibilidade do pai para a criança em função da representação retrospectiva do envolvimento do seu próprio pai

Numa primeira abordagem podemos referir que o pai interage mais com a criança ao fim-de-semana, $M=3 \mathrm{~h} 30 \mathrm{~m}, D P=2 \mathrm{~h} 40 \mathrm{~m}$, do que durante a semana, $M=1 \mathrm{~h} 21 \mathrm{~m}, D P=1 \mathrm{~h} 10 \mathrm{~m}$. Ao consideramos o Sexo da criança verificamos que, enquanto durante a semana a interacção Formas de Envolvimento x Sexo não é significativa, $F(1,311)<1$, o mesmo não se passa ao fim-de-semana, $F(1,311)=4.96, p=0,03$. De facto, ao fim-de-semana, o pai despende tempo equivalente em Interacção $(M=203.81 \mathrm{~min}$., $D P=$ 150.98 min.) e em Acessibilidade $(M=215.88 \mathrm{~min} ., D P=170.55 \mathrm{~min}$.) com uma filha, $\mathrm{F}(1,315)<1$, mas, despende mais tempo em Interacção $(M=$ 217.77 min., $D P=170.62 \mathrm{~min}$.) do que em Acessibilidade $(M=176.43$ min., $D P=153.21$ min.) com um filho, $F(1,315)=7.30, p=0,007$.

Para analisarmos em que medida a representação retrospectiva do pai relativamente ao envolvimento do seu próprio pai, ao longo da infância e adolescência, está relacionado com o tempo que este despende em Interacção e Acessibilidade com os seus próprios filhos, começamos por uma abordagem correlacional a estas variáveis.

A análise mostra que as dimensões de envolvimento apenas se correlacionam significativamente com o tempo despendido em Interacção. Concretamente, quanto mais positivamente é representado o envolvimento do próprio pai na dimensão Apoio Emocional e Estimulação e na dimensão Partilha de Tarefas, mais o adulto despende o seu tempo em Interacção com a criança (respectivamente, $r=0,24$ e 0,22 , restantes $r<0,15$ )

Para analisar o efeito da representação do envolvimento do próprio pai no tempo de envolvimento paterno com a criança, efectuámos uma categorização das dimensões da escala ENVOLVE em dois níveis, "Alto" e "Baixo", 
de acordo com a percepção retrospectiva dos pais acerca do maior ou menor envolvimento do seu próprio pai ao longo das cinco dimensões da escala. Partindo dos valores das Medianas, obtiveram-se os seguintes grupos de pais para cada dimensão: (1) AEE - Md = 2.48; "Baixo", $n=83$; "Alto", $n=79$; (2) EAS $-M d=1.44$; "Baixo", $n=74$; "Alto", $n=88$; (3) SOM $-M d=$ 3.50; "Baixo", $n=93$; "Alto", $n=69$; (4) PUN $-M d=1.13$; "Baixo", $n=$ 81; "Alto", $n=81$; (5) PT $-M d=1.00$; "Baixo", $n=89$; "Alto", $n=73$.

A análise simultânea ao efeito de cada uma das dimensões da escala ENVOLVE, categorizadas nos dois níveis acima descritos, não é comportável com a amostra disponível (o modelo ANOVA seria composto pelo total de 32 células), pelo que optámos por analisar o efeito de cada um dos factores da escala separadamente. As ANOVAs de medidas repetidas sobre Interacção e Acessibilidade, entrando cada uma das representações das formas de envolvimento do próprio pai, revelaram apenas três efeitos significativos ao longo das cinco análises. Concretamente, encontrámos um efeito principal de Nível de Envolvimento na dimensão Punição, $F(1,129)=4.42, p=0,04$, $\eta^{2}=0,03$, que indica que os pais que despendem mais tempo em Interacção e Acessibilidade, tomadas conjuntamente, são os que representam um nível de envolvimento "menos" punitivo por parte do seu próprio pai, $M=169.73$; $D P=62.67$ (nível "Alto", $M=144.58 ; D P=73.97$ ).

Quadro 1. Tempo de Interacção e Acessibilidade em função do nível de envolvimento (Alto vs. Baixo) em cada uma das dimensões da escala ENVOLVE (valores em minutos)

\begin{tabular}{lcccc}
\hline & & & Interacção & Acessibilidade \\
\hline $\begin{array}{l}\text { Apoio Emocional e } \\
\text { Estimulação }\end{array}$ & Baixo & $M(D P)$ & $143.09(79.41)$ & $165.22(99.09)$ \\
& Alto & $M(D P)$ & $173.89(92.97)$ & $147.30(79.37)$ \\
Escola e Actividades & Baixo & $M(D P)$ & $157.75(83.58)$ & $165.92(95.34)$ \\
Sociais & Alto & $M(D P)$ & $158.03(90.81)$ & $148.73(85.61)$ \\
Sustento e Orientação & Baixo & $M(D P)$ & $155.60(82.52)$ & $160.07(92.31)$ \\
Moral & Alto & $M(D P)$ & $160.98(93.63)$ & $151.96(88.03)$ \\
& Baixo & $M(D P)$ & $173.71(86.00)$ & $165.76(84.76)$ \\
Punição & Alto & $M(D P)$ & $141.85(86.18)$ & $147.31(95.26)$ \\
& Baixo & $M(D P)$ & $141.14(76.26)$ & $165.36(93.08)$ \\
Partilha de Tarefas & Alto & $M(D P)$ & $177.13(95.39)$ & $146.56(86.57)$ \\
\hline
\end{tabular}


Verificamos também uma interacção na análise relativa à representação do envolvimento do próprio pai na dimensão Apoio Emocional e Estimulação, $F(1,129)=6.61, p=0,01, \eta^{2}=0,05$. Decompondo esta interacção verifica-se que, enquanto no tempo de Acessibilidade não há diferenças significativas entre aqueles que representam o nível de envolvimento do seu pai como sendo "baixo" ou "alto", $F(1,129)=1.29, n s$, no tempo despendido em Interacção a diferença é significativa, $F(1,129)=4.18, p=0,04$ : os que consideram um nível alto de AEE dos seus próprios pais são os que despendem mais tempo em Interacção com os próprios filhos (cf. Quadro 1).

Encontramos a mesma interacção no que respeita à dimensão Partilha de Tarefas, $F(1,129)=8.45, p=0,004, \eta^{2}=0,06$. Mais uma vez, como relatado no Quadro 1, verifica-se que os dois grupos (nível alto vs. baixo) não diferem no tempo que despendem em Acessibilidade, $F(1,129)=1.42$, $n s$, mas diferem no tempo de Interacção, $F(1,129)=5.75, p=0,02$. Os pais que consideram que os seus próprios pais partilharam mais tarefas com a companheira, passam mais tempo em Interacção com a criança.

Analogamente ao que vimos apresentando, efectuámos as análises precedentes entrando na ANOVA também o Sexo da criança. Na generalidade as várias ANOVAs de medidas repetidas revelaram os efeitos reportados acima. Verificamos um efeito significativo da variável Sexo apenas na interacção Forma de Envolvimento x Nível de Envolvimento na Partilha de Tarefas x Sexo, $F(1,127)=6.00, p=0,02, \eta^{2}={ }^{\prime}, 05$. Este efeito revela que a interacção acima descrita é moderada pelo Sexo da criança. Quando a criança é do sexo feminino, não se verificam quaisquer diferenças significativas em função dos factores considerados $(\operatorname{ambos} F(1,128)<1)$. Pelo contrário, quando se trata de um rapaz, verifica-se a interacção Formas de Envolvimento $\mathrm{x}$ Nível de Envolvimento na Partilha de Tarefas, $F(1,128)=$ $14.15, p<0,001$. Isto é, quando os pais têm uma representação de um nível de Partilha de Tarefas mais "alto" do seu próprio pai com a companheira, despendem mais tempo em Interacção do que em Acessibilidade com o filho $(F(1,128)=6.25, p=0,01)$. Naqueles que representam menor nível de Partilha de Tarefas, verifica-se exactamente o inverso $(F(1,128)=4.22, p=0,04)$.

\section{Discussão e Conclusão}

Neste estudo, quando focamos a história desenvolvimental do pai, e mais concretamente nos referimos à representação acerca do envolvimento do seu próprio pai ao longo da sua infância e adolescência, encontramos um conjunto de resultados que sustenta a ideia de efeitos intergeracionais no tempo de envolvimento paterno. 
Na realidade, por um lado, verificamos que, quanto mais os pais participantes representam retrospectivamente os seus próprios pais como apoiantes e estimulantes e como homens que partilhavam as tarefas domésticas, mais tempo de envolvimento é despendido em interacção com os seus próprios filhos. No mesmo sentido, constatamos também que aqueles pais que despendem mais tempo a interagir com os filhos e estão mais disponíveis para eles (tempo em Interacção e em Acessibilidade considerado conjuntamente), são os que representam um nível de envolvimento "menos" punitivo por parte do seu próprio pai. Recordamos que ambas as dimensões se enquadram num novo papel paterno e se relacionam com as próprias mudanças nas expectativas da sociedade sobre o papel de pai (cf. Andrews et al., 2004), centrado na assumpção de um papel de educador activo e implicado no processo desenvolvimental dos filhos (e.g. Lamb, 1998). Além do mais, constata-se a relevância de uma outra forma de envolvimento paterno mais associada a um apoio prático e psicossocial à companheira, na qual muitos homens também se revêem (cf. Kelley, Smith, Green, Berndt, \& Rogers, 1998).

Por outro lado, os resultados indicam ainda que, quando os pais participantes consideram que os seus próprios pais repartiam menos as tarefas domésticas, também eles despendem menos tempo em interacção e estão menos disponíveis para os seus filhos. Encontramos aqui, provavelmente, o pontuar de um papel paterno considerado mais tradicional ligado ao sustento e à orientação moral (cf. Andrews et al., 2004; Lamb \& Tamis-LeMonda, 2004), e que é congruente com o facto de os pais participantes neste estudo terem assumido que os seus próprios pais privilegiaram um envolvimento mais centrado na dimensão do sustento económico da família e na orientação moral dos filhos.

Como se pode depreender, estes resultados realçam, particularmente, os efeitos da modelagem no comportamento paterno (cf. Palkovitz, Marks, Appleby, \& Holmes, 2003; Pleck, 1997, 2004), dando conta de uma continuidade intergeracional nas formas de envolvimento do pai com a criança, mesmo tendo em consideração a matriz multidimensional do envolvimento paterno.

Num outro nível de análise, quando explorámos o sexo da criança, enquanto factor moderador da relação entre o tempo de envolvimento despendido pelo pai em interacção e em acessibilidade com os filhos, e a representação do nível de envolvimento do próprio pai, obtivemos um outro resultado pertinente. Isto é, ao considerarmos o nível de envolvimento na partilha de tarefas, constatamos que os efeitos intergeracionais consequentes no tempo despendido em interacção e em acessibilidade com a criança, apenas se verificam em relação a um filho, não se encontrando quaisquer diferenças significativas quando a criança é do sexo feminino. 
Este resultado é consentâneo com os de vários outros estudos que sustentam que os pais se envolvem mais com os filhos do que com as filhas e, em particular, interagem mais com os primeiros (e.g. McBride, et al., 2002; Raley \& Bianchi, 2006) e mostra, uma vez mais, como o sexo da criança pode influenciar, designadamente, as expectativas e o comportamento do pai em relação à criança (Jacobs \& Bleeker, 2004).

Em suma, o conjunto de resultados deste estudo reforça a importância do envolvimento paterno no processo desenvolvimental da criança, nomeadamente, com os rapazes, podendo-se descortinar efeitos ao nível de um impacto intergeracional na forma como os pais contemporâneos despendem o seu tempo de envolvimento com os filhos, uma vez que os seus próprios pais serviram de modelo a esse mesmo envolvimento.

Finalmente, podemos identificar algumas limitações neste estudo, a maioria das quais decorrente da natureza exploratória deste trabalho e do facto de fazer parte de uma investigação mais alargada acerca das formas, factores e consequências do envolvimento do pai no processo desenvolvimental da criança em idade escolar. De modo específico, salientamos, a necessidade de melhorar as qualidades psicométricas da escala ENVOLVE; a relevância de obter informação sobre a qualidade do envolvimento entre o pai e a criança (para além dos dados de natureza mais quantitativa obtidos a partir do tempo de envolvimento paterno em interacção e em acessibilidade); e a importância de conservar a coerência conceptual com o modelo tripartido de envolvimento paterno (cf. Lamb et al., 1987), incluindo na investigação dados relativos à assumpção de responsabilidades pelo pai.

\section{Referências}

Ahlberg, C., \& Sandnabba, K. (1998). Parental nurturance and identification with own father and mother: The reproduction of nurturant parenting. Early Development and Parenting, 7, 211-221.

Andrews, A. B., Luckey, I., Bolden, E., Whiting-Fickling, J., \& Lind, K. A. (2004). Public perceptions about father involvement. Journal of Family Issues, 25(5), 603-633.

Camus, J. L., \& Frascarolo, F. (2003). Introduction of the special issue on fatherhood. European Journal of Psychology of Education, 28(2), 95-99.

Fuligni, A., \& Brooks-Gunn, J. (2004). Measuring mother and father shared caregiving: An analysis using the Panel Study of Income Dynamics-Child Development Supplement. In R. D. Day \& M. E. Lamb (Eds.), Conceptualizing and measuring father involvement (pp. 341-357). Mahwah, New Jersey: Lawrence Erlbaum Associates. 
Hawkins, A., Bradford, K. P., Palkovitz, R., Christiansen, S. L., Day, R. D., \& Call, V. R. A. (2002). The inventory of father involvement: A pilot study of a new measure of father involvement. The Journal of Men's Studies, 10(2), 183-196.

Hofferth, S. L., \& Sandberg, J. (2001). How American children spend their time. Journal of Marriage and Family, 63(2), 295-308.

Jacobs, J. E., \& Bleeker, M. M. (2004). Girls' and boys' developing interests in math and science: Do parents matter? New Directions for Child and Adolescent Development, 106, 5-21.

Lamb, M. E. (1998). Fatherhood then and now. In A. Booth \& A. C. Crouter (Eds.), Men in families: When do they get involved? What difference does it make? (pp. 47-52). Mahwah, New Jersey: Lawrence Erlbaum Associates.

Lamb, M. E. (2000). The history of research on father involvement: An overview. Marriage \& Family Review, 29, 23-42.

Lamb, M. E., Pleck, Charnov, E. L., \& Levine, J. A. (1987). A biosocial perspective on paternal behaviour and involvement. In J. B. Lancaster, J. Altman, A. Rossi \& L. R. Sherrod (Eds.), Parenting across the lifespan: Biosocial perspectives (pp. 11-42). New York: Academic.

Lima, J. A. (2009). O envolvimento do pai no processo desenvolvimental da criança em idade escolar: Formas, factores e consequências. Tese de Doutoramento. Porto: FPCE-UP.

Lima, J. A., Serôdio, R. G., \& Cruz, O. (2008). Filho és, pai serás...: A percepção retrospectiva do pai acerca das formas de envolvimento do seu próprio pai e suas consequências desenvolvimentais. International Journal of Developmental and Educational Psychology (INFAD), 4(1), 101-112.

Losh-Hasselbart, S. (1987). The development of gender roles. In M. B. Sussman \& S. Steinmetz (Eds.), Handbook of marriage and the family (pp. 535-563). New York: Plenum.

Kelley, M. L., Smith, T., Green, A., Berndt, A., \& Rogers, M. (1998). Importance of father's parenting to African-American toddler's social and cognitive development. Infant Behaviour \& Development, 21(4), 733-744.

Marsiglio, W., Amato, P. R., Day, R. D., \& Lamb, M. E. (2000). Scholarship on fatherhood in the 1990s and beyond. Journal of Marriage and Family, 62(4), 1173-1191.

Marsiglio, W., Hutchinson, S., \& Cohan, M. (2000). Envisioning fatherhood: A social psychological perspective on young men without kids. Family Relations, 49(2), 133-142.

McBride, B. A., Schoppe, S. J., \& Rane, T. R. (2002). Child characteristics, parenting stress, and parental involvement: Fathers versus mothers. Journal of Marriage and Family, 64(4), 998-1011.

Palkovitz, R., Marks, L., Appleby, D., \& Holmes, E. K. (2003). Parenting and adult development: Contexts, processes and products of intergenerational relationships. In L. Kuczynski (Ed.), Dynamics in parent-child relations (pp. 307-323). Thousand Oaks, CA: Sage. 
Parke, R. D. (1995). Fathers and families. In M. H. Bornstein (Ed.), Handbook of parenting (Vol. 3, pp. 27-63). Mahwah, New Jersey: Lawrence Erlbaum Associates.

Parke, R. D. (2000). Father involvement: A developmental psychological perspective. Marriage \& Family Review, 29(2-3), 43-58.

Pleck, J. (1997). Paternal involvement: Levels, sources, and consequences. In M. E. Lamb (Ed.), The role of the father in child development $\left(3^{\text {rd }}\right.$ ed., pp. 66-103). New York: John Wiley \& Sons.

Pleck, J. \& Masciadrelli, B. P. (2004). Paternal involvement by U. S. residential fathers: Levels, sources and consequences. In M. E. Lamb (Ed.), The role of the father in child development ( $4^{\text {th }}$ ed., pp. 222-270). New York: John Wiley $\&$ Sons.

Raley, S., \& Bianchi, S. (2006). Sons, daughters, and family processes: Does gender of children matter? Annual Review of Sociology, 32, 401-421.

Volling, B. L., \& Belsky, J. (1992). The contribution of mother-child and father-child relationships to the quality of sibling interaction: A longitudinal study. Child Development, 63(5), 1209-1222.

Wood, J. J., \& Repetti, R. L. (2004). What gets dad involved? A longitudinal study of change in parental child caregiving involvement. Journal of Family Psychology, 18(1), 237-249. 